\title{
Article \\ BRCA 1/2 Germline Mutation Predicts the Treatment Response of FOLFIRINOX with Pancreatic Ductal Adenocarcinoma in Korean Patients
}

\author{
Ji Hoon Park ${ }^{1}\left(\mathbb{D}\right.$, Jung Hyun Jo ${ }^{1}\left(\mathbb{D}\right.$, Sung Ill Jang ${ }^{2} \mathbb{C}$, Moon Jae Chung ${ }^{1} \mathbb{D}$, Jeong Youp Park ${ }^{1}$, Seungmin Bang ${ }^{1}$, \\ Seung Woo Park ${ }^{1}$, Si Young Song ${ }^{1}$, Hee Seung Lee ${ }^{1, * \mathbb{D}}$ and Jae Hee Cho ${ }^{2, *(\mathbb{D})}$
}

Citation: Park, J.H.; Jo, J.H.; Jang, S.I.; Chung, M.J.; Park, J.Y.; Bang, S.;

Park, S.W.; Song, S.Y.; Lee, H.S.;

Cho, J.H. BRCA $1 / 2$ Germline

Mutation Predicts the Treatment

Response of FOLFIRINOX with

Pancreatic Ductal Adenocarcinoma in

Korean Patients. Cancers 2022, 14, 236.

https://doi.org/10.3390/

cancers14010236

Academic Editors: Vincenza Barresi and Robert C. Bast, Jr.

Received: 2 November 2021

Accepted: 28 December 2021

Published: 4 January 2022

Publisher's Note: MDPI stays neutral with regard to jurisdictional claims in published maps and institutional affiliations.

Copyright: (c) 2022 by the authors. Licensee MDPI, Basel, Switzerland. This article is an open access article distributed under the terms and conditions of the Creative Commons Attribution (CC BY) license (https:// creativecommons.org/licenses/by/ $4.0 /)$.
1 Department of Internal Medicine, Division of Gastroenterology, Yonsei University College of Medicine, Seoul 03722, Korea; jihoon815@yuhs.ac (J.H.P.); JUNGHYUNJO83@yuhs.ac (J.H.J.);

MJCHUNG@yuhs.ac (M.J.C.); sensass@yuhs.ac (J.Y.P.); bang7028@yuhs.ac (S.B.); swoopark@yuhs.ac (S.W.P.); sysong@yuhs.ac (S.Y.S.)

2 Department of Internal Medicine, Gangnam Severance Hospital, Yonsei University College of Medicine, Seoul 06273, Korea; aerojsi@yuhs.ac

* Correspondence: lhs6865@yuhs.ac (H.S.L.); jhcho9328@yuhs.ac (J.H.C.); Tel.: +82-2-2228-1935 (H.S.L.); +82-2-2019-3310 (J.H.C.)

Simple Summary: In pancreatic ductal adenocarcinoma, FOLFIRINOX and nab-paclitaxel are recommended as first-line chemotherapy regimens. However, there are limited data to predict the efficacy of the FOLFIRINOX regimen in patient outcomes. Platinum-based chemotherapy is tolerable and responsible in patients with DNA damage repair gene mutations. However, data are still limited, and no Asian data are available yet. Here, we sought to investigate the proportion of germline BRCA $1 / 2$ mutations in patients with germline blood tests. Finally, we investigated the treatment response of FOLFIRINOX in patients with BRCA 1/2 mutations. We found that the presence of germline $B R C A 1 / 2$ mutations was associated with an improved overall response rate in pancreatic ductal adenocarcinoma patients treated with FOLFIRINOX. The high response rate in this analysis supports the preferential use of FOLFIRINOX therapy for patients harboring a BRCA germline mutation, and supports the need for early germline testing in order to select the best therapy.

Abstract: We evaluated the proportion of BRCA 1/2 germline mutations in Korean patients with sporadic pancreatic ductal adenocarcinoma (PDAC) and its effect on the chemotherapeutic response of FOLFIRINOX. This retrospective study included patients who were treated at two tertiary hospitals between 2012 and 2020, were pathologically confirmed to have PDAC, and had undergone targeted next-generation sequencing-based germline genetic testing. Sixty-six patients were included in the study (24 men; median age 57.5 years). In the germline test, $B R C A 1 / 2$ pathogenic mutations were found in nine patients $(9 / 66,13 \%, B R C A 1, n=3 ; B R C A 2, n=5$; and $B R C A 1 / 2, n=1)$. There was no significant difference in the baseline characteristics according to $B R C A$ mutation positivity. Among patients who underwent FOLFIRINOX chemotherapy, patients with a BRCA 1/2 mutation showed a higher overall response rate than those without a BRCA $1 / 2$ mutation $(71.4 \%$ vs. $13.9 \%, p=0.004)$. Patients with a germline $B R C A 1 / 2$ mutation showed longer progression-free survival than those without a $B R C A 1 / 2$ mutation, without a significant time difference (18 months vs. 10 months, $p=0.297)$. Patients with a $B R C A 1 / 2$ mutation in the germline blood test had a higher response rate to FOLFIRINOX chemotherapy in PDAC. The high proportion of BRCA $1 / 2$ germline mutations and response rate supports the need for germline testing in order to predict better treatment response.

Keywords: breast cancer gene; BRCA; FOLFIRINOX; pancreatic ductal adenocarcinoma

\section{Introduction}

Pancreatic ductal adenocarcinoma (PDAC) is expected to become the second leading cause of cancer-related deaths in the US before 2030 [1]. In the NCCN (National Com- 
prehensive Cancer Network) guidelines, germline testing is recommended for patients with PDAC, using comprehensive gene panels for hereditary cancer syndromes [2]. The genes commonly associated with pathogenic germline alterations are BRCA 1/2, ATM, PALB2, MLH1, MSH2, MSH6, PMS2, CDKN2A, and TP53 [3]. Among them, the frequency of detected BRCA $1 / 2$ (breast cancer susceptibility gene- 1 and -2 ) is $4 \%$ to $7 \%[4,5]$. The risk for pancreatic cancer is elevated two- to six-fold in these patients [6,7].

Recently, the POLO trial showed the benefit of poly ADP ribose polymerase (PARP) inhibitors in BRCA mutations. BRCA genes encode proteins involved in homologous recombination repair, and cells with mutations are sensitive to PARP inhibitors. However, there was no difference in overall survival between the PARP inhibitor and placebo groups $(p=0.68)$ [8]. Furthermore, in the real world, it is difficult for clinicians to change regimens in patients who are tolerant to FOLFIRINOX (oxaliplatin, irinotecan, folinic acid, and fluorouracil) chemotherapy.

In PDAC, FOLFIRINOX and nab-paclitaxel are recommended as first-line chemotherapy regimens. The guidelines recommend FOLFIRINOX in patients who are young and with better performance status (ECOG 0-1) [2]. However, there are limited data to predict the efficacy of the FOLFIRINOX regimen in patient outcomes [9]. Platinum-based chemotherapy is tolerable and responsible in patients with DNA damage repair gene mutations $[10,11]$. However, data are still limited, and no Asian data are available yet [12-16].

Here, we sought to investigate the proportion of germline BRCA 1/2 mutations in patients with germline blood tests. Finally, we investigated the treatment response of FOLFIRINOX in patients with a BRCA $1 / 2$ mutation.

\section{Materials and Methods}

\subsection{Study Population}

This dual institutional retrospective analysis was performed on all patients diagnosed with PDAC who underwent a germline blood test between January 2012 and February 2020. We identified 66 patients who underwent a germline blood test. Of these, two patients were excluded from the study on account of insufficient clinical data $(n=2)$. One patient was diagnosed and treated at another hospital, and one patient died shortly after diagnosis due to deterioration of the condition. The remaining 64 patients were included in the analysis. This study was performed in accordance with the Declaration of Helsinki, as reflected by the institutional review board of Severance Hospital (approval number 4-2021-1151).

\subsection{Variables}

We evaluated patient characteristics, laboratory variables, tumor characteristics, progressionfree survival (PFS), overall survival (OS), and overall response rate (ORR). Patient demographics and clinical characteristics, including age, sex, personal and family history of cancer, hypertension, diabetes mellitus, smoking history, body mass index (BMI), systemic chemotherapy, and response to treatment, were obtained from medical records and imaging studies. BMI, defined as body weight divided by the square of the height, was categorized following the guidelines of the World Health Organization (WHO 2000) (BMI < 18.5, underweight; $18.5-24.9$, normal range; $\geq 25.0$, overweight; and $\geq 30.0$, obese). Tumor characteristics (location, extent, and number of metastatic organs) and laboratory characteristics (carbohydrate antigen [CA] 19-9) were also investigated.

The date of death and the date of the last follow-up were reviewed to estimate the OS and PFS. We observed both survival and follow-up data until 5 March 2021. OS was defined as the interval from the start of FOLFIRINOX until death. PFS was defined as the interval from the start of FOLFIRINOX to progressive disease (PD) or death. Patients who remained without death or PD were censored at the time of the last follow-up. Responses were determined using RECIST (response evaluation criteria in solid tumors) v1.1. ORR was defined as the percentage of patients who had a best response rating of complete response (CR) or partial response (PR) at any time point during treatment with chemotherapy. Patients without measurable disease at baseline were excluded from the ORR analysis. 


\subsection{DNA Extraction and Sequencing}

Genomic DNA was extracted from peripheral blood using a QIAamp DNA Blood Mini Kit (Qiagen, Venlo, The Netherlands). The amount of input DNA was approximately 500 ng. DNA was fragmented into segments between 150 and 250 bp using the Bioruptor ${ }^{\circledR}$ Pico sonication system (Diagenode, Liege, Belgium), end-repaired, and ligated to Illumina adapters (Illumina, San Diego, CA, USA) and indices. Sequencing libraries were hybridized with capture probes (Celemic, Seoul, Korea). The enriched DNA was then amplified, and clusters were generated and sequenced on a NextSeq 550 instrument (Illumina) with $2 \times 151$ bp reads [17]. Pathogenicity interpretations of the variants were performed according to the 2015 American College of Medical Genetics and Genomics guidelines by professional medical geneticists, using evidence from variant type assessments, population allele frequency, prediction algorithm results, and searches within databases such as ClinVar.

\subsection{Statistical Analysis}

The baseline demographics and characteristics of the patients were analyzed using descriptive statistics. The differences in baseline characteristics and ORR between BRCApositive and $B R C A$-negative groups were analyzed using the chi-square test for categorical variables and the Student's $t$-test for continuous variables. We estimated the median OS and PFS according to BRCA mutations using Kaplan-Meier curves and compared them using the log-rank test. A time-dependent Cox regression analysis was applied to estimate hazard ratios (HRs) with 95\% confidence intervals (CIs) of pancreatic cancer mortality associated with $B R C A$ mutations. Statistical significance was set at $p<0.05$. All analyses were conducted using SPSS version 26.0 (SPSS, Chicago, IL, USA).

\section{Results}

\subsection{Patients' Characteristics and BRCA 1/2 Gene Mutations}

A total of 66 PDAC patients underwent germline mutation analysis. Of all participants, three patients $(4.5 \%)$ had a $B R C A 1$ mutation, five $(7.6 \%)$ had a $B R C A 2$ mutation, and one patient $(1.5 \%)$ had a BRCA $1 / 2$ mutation. None had germline ATM or PALB2 mutations. Two patients had KRAS mutations, and one patient each had TP53, CDK2NA, SMAD4, and MUTYH mutations. Ten patients (15.2\%) had BRCA variants of unknown significance (VUS). The specific BRCA mutations are listed in Supplementary Table S1. During the study period, somatic mutation tests were performed on 31 patients not included in this study. Two patients $(2 / 31,6.5 \%)$ had a BRCA1 mutation, and two patients $(2 / 31,6.5 \%)$ had a BRCA2 mutation. They were not included in this analysis. Of these 66 patients, 2 patients were excluded due to insufficient clinical data. Of these 64 patients, 7 patients had resectable PDAC, 20 patients had borderline resectable or locally advanced PDAC, and 37 patients had metastatic PDAC. Seven patients with resectable PDAC underwent curative intent resection (Figure 1). This study was performed in accordance with the Declaration of Helsinki, as reflected by the institutional review board of Severance Hospital (approval number 4-2021-1151).

The patient demographics and clinical characteristics are summarized in Table 1 . The median age of the patients was 57.5 years (interquartile range, $48.0-66.8$ years), and $37.5 \%$ were men. Overall, $29.7 \%(19 / 64)$ of the patients in our study also had a personal history of malignancy, including breast cancer $(6 / 64,9.4 \%)$, thyroid cancer $(2 / 64,3.1 \%)$, and ovarian cancer $(1 / 64,1.6 \%)$. A higher percentage of patients in the BRCA-positive group had a prior history of malignancy $(6 / 9,66.7 \%$ vs. $13 / 55,23.6 \%, p=0.016)$ and breast cancer $(4 / 9,44.4 \%$ vs. $2 / 55,3.64 \%)$. Of the 20 patients who had a family history of cancer, 10 $(22.7 \%)$ were of pancreatic cancer, $3(6.8 \%)$ were of breast cancer, and $12(27.3 \%)$ were of other malignancies. It was not possible to identify whether the family history of cancer was from a first-degree relative or not. The proportion of family history of any malignancy and number of metastatic sites was greater in the $B R C A$-positive group than in the $B R C A$ negative group; however, the difference was not significant (all, $p>0.05$ ). The other 
variables showed no significant differences between the two groups. In the Kaplan-Meier survival analysis, there was no significant difference seen in OS between the $B R C A$-positive and $B R C A$-negative groups $(p=0.888$ ) (Supplementary Figure S1). The multivariable Cox regression model showed no significant improvement in OS in the presence of $B R C A 1 / 2$ mutations (HR, 0.128; 95\% CI, 0.021-1.618) (Supplementary Table S2). The risk factors related to OS were tumor location (HR, 7.335; 95\% CI, 2.030-26.503, $p=0.002)$, T stage (HR, 0.333; 95\% CI, 0.115-0.963, $p=0.042)$, and M stage (HR, 7.661; 95\% CI, 2.188-26.824, $p=0.001)$ in the multivariate analysis.

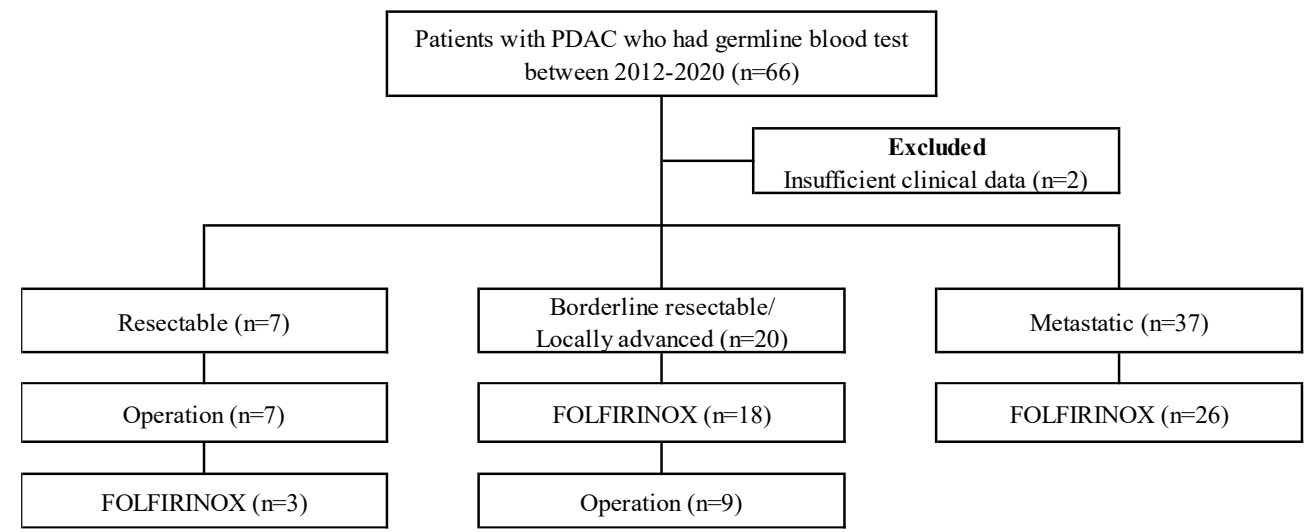

Figure 1. Selection of study population with PDAC, pancreatic ductal adenocarcinoma; FOLFIRINOX: oxaliplatin, irinotecan, folinic acid, and fluorouracil.

Table 1. Baseline characteristics of patients who had germline genetic blood tests.

\begin{tabular}{|c|c|c|c|c|}
\hline Variables & Total $(n=64)$ & $\begin{array}{c}\text { BRCA Mutation } \\
(+) \\
(n=9)\end{array}$ & $\begin{array}{c}\text { BRCA Mutation } \\
(-) \\
(n=55)\end{array}$ & $p$ Value \\
\hline Age at diagnosis (year) & $57.5(48.0-66.8)$ & $50.0(47.0-60.0)$ & $59.0(48.0-71.0)$ & 0.122 \\
\hline Male & $24(37.5 \%)$ & $2(22.2 \%)$ & $22(40.0 \%)$ & 0.464 \\
\hline \multicolumn{5}{|l|}{$\begin{array}{l}\text { History of prior } \\
\text { malignancy, } n(\%)\end{array}$} \\
\hline Yes & $19(29.7 \%)$ & $6(66.7 \%)$ & $13(23.6 \%)$ & 0.016 \\
\hline Breast & $6(9.4 \%)$ & $4(44.4 \%)$ & $2(3.64 \%)$ & 0.014 \\
\hline \multicolumn{5}{|l|}{$\begin{array}{c}\text { Family history of any } \\
\text { malignancy, } n(\%)\end{array}$} \\
\hline Yes & $29(45.3 \%)$ & $6(66.7 \%)$ & $23(41.8 \%)$ & 0.279 \\
\hline Pancreas & $14(21.9 \%)$ & $2(22.2 \%)$ & $12(21.8 \%)$ & 1.000 \\
\hline Breast & $3(4.7 \%)$ & $1(11.1 \%)$ & $2(3.6 \%)$ & 1.000 \\
\hline $\begin{array}{c}\text { Tobacco use }(\%) \\
\text { Yes (past or current) } \\
\text { BMI }\left(\mathrm{kg} / \mathrm{m}^{2}\right)\end{array}$ & $16(36.4 \%)$ & $3(33.3 \%)$ & $19(34.5 \%)$ & 1.000 \\
\hline$\geq 25.0$ & $9(14.1 \%)$ & $1(11.1 \%)$ & $8(14.5 \%)$ & 1.000 \\
\hline Diabetes Mellitus & $18(28.1 \%)$ & $1(11.1 \%)$ & $17(30.9 \%)$ & 0.425 \\
\hline $\begin{array}{l}\text { Hypertension } \\
\text { CA 19-9 (U/mL) }\end{array}$ & $21(32.8 \%)$ & $2(22.2 \%)$ & $19(34.5 \%)$ & 0.706 \\
\hline $\begin{array}{c}\text { Elevated }(>34.0 \mathrm{U} / \mathrm{mL}) \\
\text { Pathology }\end{array}$ & $51(79.7 \%)$ & $8(88.9 \%)$ & $43(78.2 \%)$ & 0.672 \\
\hline Well-differentiated & $2(3.1 \%)$ & $0(0.0 \%)$ & $2(3.6 \%)$ & \\
\hline Moderately differentiated & $24(37.5 \%)$ & $7(77.8 \%)$ & $17(30.9 \%)$ & \\
\hline $\begin{array}{l}\text { Poorly differentiated } \\
\text { Clinical T stage }\end{array}$ & $7(10.9 \%)$ & $2(22.2 \%)$ & $5(9.1 \%)$ & \\
\hline
\end{tabular}


Table 1. Cont.

\begin{tabular}{|c|c|c|c|c|}
\hline Variables & Total $(n=64)$ & $\begin{array}{c}\text { BRCA Mutation } \\
(+) \\
(n=9)\end{array}$ & $\begin{array}{c}\text { BRCA Mutation } \\
(-) \\
(n=55)\end{array}$ & $p$ Value \\
\hline $\mathrm{T} 1 / 2$ & $25(39.1 \%)$ & $3(33.3 \%)$ & $22(40.0 \%)$ & 1.000 \\
\hline $\mathrm{T} 3 / 4$ & $39(60.9 \%)$ & $6(66.7 \%)$ & $33(60.0 \%)$ & \\
\hline \multicolumn{5}{|l|}{ Clinical $n$ stage } \\
\hline No & $26(40.6 \%)$ & $5(55.6 \%)$ & $21(38.2 \%)$ & 0.467 \\
\hline \multicolumn{5}{|c|}{ Location of primary tumor } \\
\hline Head & $32(50.0 \%)$ & $4(44.4 \%)$ & $28(50.9 \%)$ & 1.000 \\
\hline \multicolumn{5}{|l|}{ Metastasis site } \\
\hline Liver & $22(34.4 \%)$ & $6(66.7 \%)$ & $16(29.1 \%)$ & 0.053 \\
\hline Peritoneum & $13(20.3 \%)$ & $0(0.0 \%)$ & $13(23.6 \%)$ & 0.185 \\
\hline Distant LN & $9(14.1 \%)$ & $1(11.1 \%)$ & $8(14.5 \%)$ & 1.000 \\
\hline \multicolumn{5}{|c|}{ Number of metastasis site } \\
\hline 0 site & $33(51.6 \%)$ & $3(33.3 \%)$ & $30(54.5 \%)$ & 0.296 \\
\hline 1 or more sites & $31(48.4 \%)$ & $6(66.7 \%)$ & $25(45.5 \%)$ & \\
\hline
\end{tabular}

Data are in $\mathrm{n}(\%)$ or median (IQR). BRCA: breast cancer susceptibility gene; BMI: body mass index; CA: carbohydrate antigen; LN: lymph node; IQR: interquartile range.

\subsection{FOLFIRINOX Treatment and Overall Response Rate}

In total, 47 patients of the study participants received FOLFIRINOX chemotherapy. Of these 47 patients, 4 had no response evaluation and were hence excluded from the ORR analysis. The patient demographics and clinical characteristics are summarized in Table 2. Of these 43 patients, $7(16.3 \%)$ had a BRCA mutation. The median age of the patients was 51.0 years (interquartile range, $46.0-65.0$ years), and $41.9 \%$ were male. Of the $B R C A$-positive group, $57.1 \%(4 / 7)$ had a history of prior malignancy, compared with $16.7 \%(6 / 36)$ of the $B R C A$-negative group, and there was a significant difference observed between the groups $(p=0.040)$. A history of breast cancer was reported in $42.9 \%(3 / 7)$ and $2 \%(2 / 36)$ of the $B R C A$-positive and $B R C A$-negative groups, respectively $(p=0.024)$. A median of 12.0 FOLFIRINOX cycles were administered to the BRCA-positive patients, and 9.0 cycles were administered to the $B R C A$-negative patients. FOLFIRINOX therapy was mostly administered in the first-line setting: $93.0 \%$ (40/43) in the first-line setting and $7.0 \%(3 / 43)$ in the second-line setting. The ORR, as defined by RECIST v1.1, was significantly higher in BRCA-positive patients than in BRCA-negative patients $(5 / 7,71.4 \%$ vs. $5 / 36,13.9 \% ; p=0.004$ ) (Table 3). For $B R C A$-positive patients, partial response and stable disease were observed in $71.4 \%(5 / 7)$ and $28.6 \%(2 / 7)$ of patients, respectively. None of the $B R C A$-positive patients showed a complete response. Of the 43 patients, $7(16.3 \%)$ had a $B R C A$ mutation and $5(11.6 \%)$ had a $B R C A$ mutation of unknown significance. The ORR was significantly higher in $B R C A$-positive patients, including those with mutations of unknown significance, than in $B R C A$-negative patients $(7 / 12,58.3 \%$ vs. $3 / 31,9.7 \%$; $p=0.002$ ) (Supplementary Table S3). A subset analysis was performed to test the effect of gemcitabine/nab-paclitaxel on the ORR. Patients with a BRCA $1 / 2$ mutation did not show a significantly better response than those without a $B R C A 1 / 2$ mutation $(1 / 3,33.3 \%$ vs. $0 / 17,0.0 \%, p=0.154)$. 
Table 2. Baseline characteristics of the patients who received FOLFIRINOX treatment and had response evaluation results.

\begin{tabular}{|c|c|c|c|c|}
\hline Variables & Total $(n=43)$ & $\begin{array}{c}\text { BRCA Mutation } \\
(+) \\
(n=7)\end{array}$ & $\begin{array}{c}\text { BRCA Mutation } \\
(-) \\
(n=36)\end{array}$ & $p$ Value \\
\hline Age at diagnosis (year) & $51.0(46.0-65.0)$ & $49.0(46.0-56.0)$ & $51.5(44.5-65.8)$ & 0.508 \\
\hline Male & $18(41.9 \%)$ & $2(28.6 \%)$ & $16(44.4 \%)$ & 0.680 \\
\hline \multicolumn{5}{|l|}{$\begin{array}{l}\text { History of prior } \\
\text { malignancy, } n(\%)\end{array}$} \\
\hline Yes & $10(23.3 \%)$ & $4(57.1 \%)$ & $6(16.7 \%)$ & 0.040 \\
\hline Breast & $5(11.6 \%)$ & $3(42.9 \%)$ & $2(5.6 \%)$ & 0.024 \\
\hline \multicolumn{5}{|l|}{$\begin{array}{c}\text { Family history of any } \\
\text { malignancy, } n(\%)\end{array}$} \\
\hline Yes & $22(51.2 \%)$ & $6(85.7 \%)$ & $16(44.4 \%)$ & 0.095 \\
\hline Pancreas & $10(23.3 \%)$ & $2(28.6 \%)$ & $8(22.2 \%)$ & 0.656 \\
\hline Breast & $3(7.0 \%)$ & $1(14.3 \%)$ & $2(5.6 \%)$ & 0.421 \\
\hline \multicolumn{5}{|l|}{ Tobacco use (\%) } \\
\hline $\begin{array}{l}\text { Yes (past or current) } \\
\text { BMI }\left(\mathrm{kg} / \mathrm{m}^{2}\right)\end{array}$ & $15(34.9 \%)$ & $3(42.9 \%)$ & $12(33.3 \%)$ & 0.680 \\
\hline$\geq 25.0$ & $9(20.9 \%)$ & $1(14.3 \%)$ & $5(13.9 \%)$ & 1.000 \\
\hline Diabetes Mellitus & $9(20.9 \%)$ & $0(0.0 \%)$ & $9(25.0 \%)$ & 0.314 \\
\hline Hypertension & $11(25.6 \%)$ & $2(28.6 \%)$ & $9(25.0 \%)$ & 1.000 \\
\hline \multicolumn{5}{|l|}{ CA $19-9(\mathrm{U} / \mathrm{mL})$} \\
\hline \multicolumn{4}{|l|}{ Pathology } & 1.000 \\
\hline Well-differentiated & $1(2.3 \%)$ & $0(0.0 \%)$ & $1(2.8 \%)$ & \\
\hline $\begin{array}{l}\text { Moderately } \\
\text { differentiated }\end{array}$ & $16(37.2 \%)$ & $5(71.4 \%)$ & $11(30.6 \%)$ & \\
\hline \multicolumn{4}{|l|}{ Clinical T stage } & \\
\hline $\mathrm{T} 1 / 2$ & $16(37.2 \%)$ & $2(28.6 \%)$ & $14(38.9 \%)$ & 0.695 \\
\hline $\mathrm{T} 3 / 4$ & $27(62.8 \%)$ & $5(71.4 \%)$ & $22(61.1 \%)$ & \\
\hline \multicolumn{5}{|l|}{ Clinical $n$ stage } \\
\hline No & $16(37.2 \%)$ & $3(42.9 \%)$ & $13(36.1 \%)$ & 1.000 \\
\hline \multicolumn{5}{|l|}{$\begin{array}{l}\text { Location of primary } \\
\text { tumor }\end{array}$} \\
\hline Head & $22(51.2 \%)$ & $3(42.9 \%)$ & $19(52.8 \%)$ & 0.698 \\
\hline \multicolumn{5}{|l|}{ Metastasis site } \\
\hline Liver & $17(39.5 \%)$ & $5(71.4 \%)$ & $12(33.3 \%)$ & 0.093 \\
\hline Peritoneum & $8(18.6 \%)$ & $0(0.0 \%)$ & $8(22.2 \%)$ & 0.315 \\
\hline Distant LN & $5(11.6 \%)$ & $1(14.3 \%)$ & $4(11.1 \%)$ & 1.000 \\
\hline \multicolumn{5}{|l|}{$\begin{array}{c}\text { Number of metastasis } \\
\text { site }\end{array}$} \\
\hline 0 site & $19(44.2 \%)$ & $2(28.6 \%)$ & $17(47.2 \%)$ & 0.243 \\
\hline 1 or more sites & $24(55.8 \%)$ & $5(71.4 \%)$ & $19(52.8 \%)$ & \\
\hline
\end{tabular}


Table 3. Overall response rate to FOLFIRINOX in patients with a germline BRCA $1 / 2$ mutation was significantly higher than patients without the mutation.

\begin{tabular}{cccc}
\hline Outcome & $\begin{array}{c}\text { BRCA Mutation } \\
(+) \\
(n=7)\end{array}$ & $\begin{array}{c}\text { BRCA Mutation } \\
(-) \\
(n=36)\end{array}$ & $p$ Value \\
\hline Overall response rate, $n(\%)$ & $5(71.4 \%)$ & $5(13.9 \%)$ & 0.004 \\
Complete response & 0 & 0 & \\
Partial response & $5(71.4 \%)$ & $5(13.9 \%)$ & \\
Stable disease & $2(28.6 \%)$ & $26(72.2 \%)$ & \\
Progressive disease & 0 & $5(13.9 \%)$ & 0.421 \\
Line of FOLFIRINOX therapy & & $34(94.4 \%)$ & \\
First & $6(85.7 \%)$ & $2(5.6 \%)$ & \\
Second & $1(14.3 \%)$ &
\end{tabular}

Response criteria according to RECIST (response evaluation criteria in solid tumours) v1.1. FOLFIRINOX oxaliplatin, irinotecan, folinic acid, and fluorouracil; BRCA: breast cancer susceptibility gene.

\subsection{Progression-Free Survival and Overall Survival}

In our study, $71.2 \%(47 / 66)$ of the patients received FOLFIRINOX treatment. Of the 47 patients, 32 patients with locally advanced, metastatic, or recurrent PDAC were treated with palliative first-line FOLFIRINOX. These cases were included in the survival analyses. As a clinically relevant surrogate of the durability of FOLFIRINOX responses, we utilized PFS, defined as the date of first FOLFIRINOX chemotherapy administration to the date of clinical treatment failure. BRCA-positive patients had longer PFS than BRCA-negative patients. However, this association was not significant $(p=0.423)$. The median PFS was 18.0 months for $B R C A$-positive patients and 10.0 months for $B R C A$-negative patients. The OS for $B R C A$-positive and $B R C A$-negative patients did not reach the median (Figure 2).
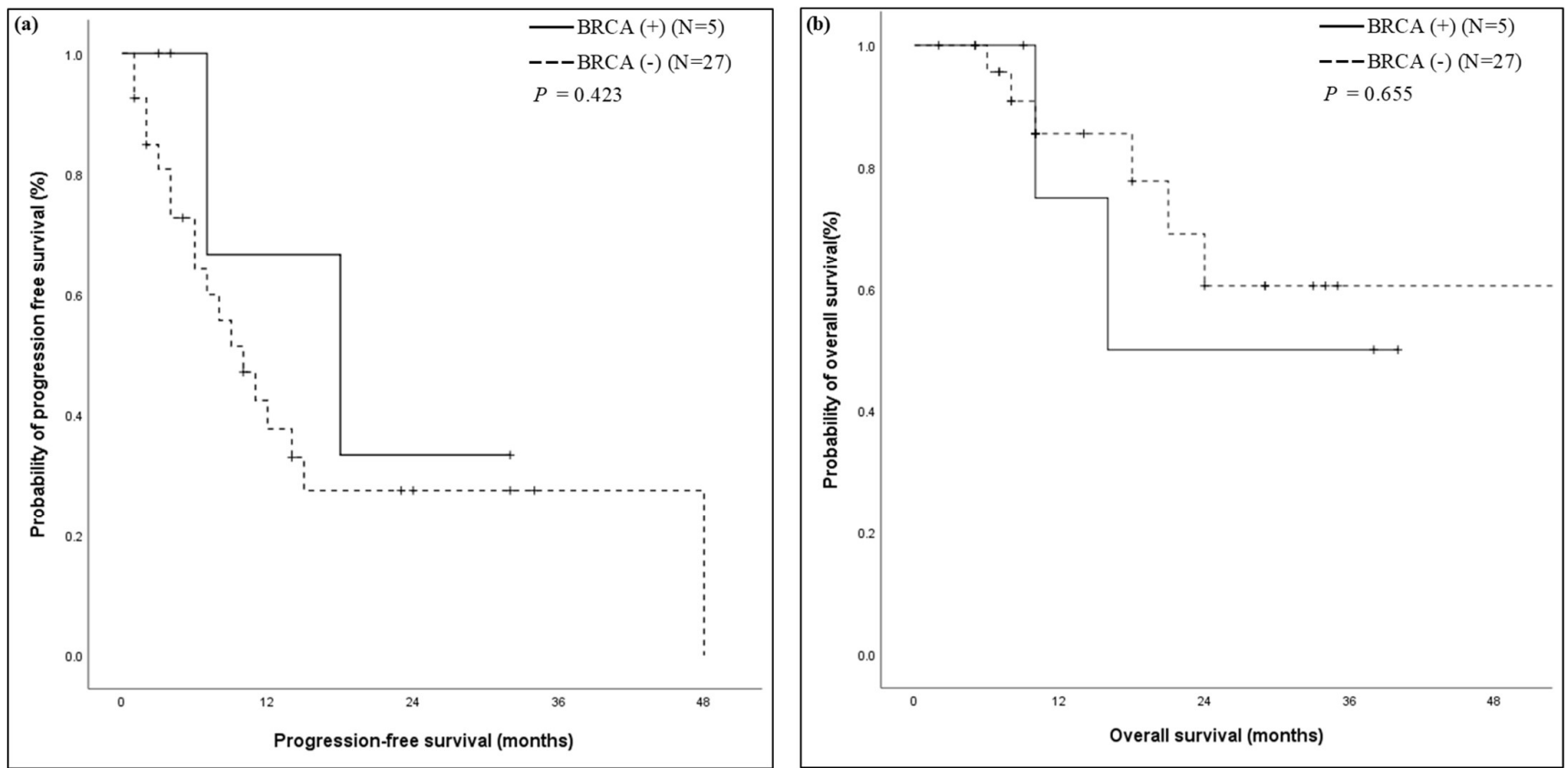

Figure 2. Kaplan-Meier curve in patients with first-line FOLFIRINOX stratified by the presence of a germline BRCA gene mutation. (a) The PFS was 18 months (95\% confidence interval 0.4-35.6) in the $B R C A$-positive group, as compared to 10 months (95\% confidence interval 5.5-14.5) in the $B R C A$-negative group $(p=0.423)$; (b) Kaplan-Meier survival curves for overall survival of the patients were not reached. FOLFIRINOX: oxaliplatin, irinotecan, folinic acid, and fluorouracil; BRCA: breast cancer susceptibility gene. 


\section{Discussion}

In this study, $B R C A 1 / 2$ germline mutations predicted the treatment response of FOLFIRINOX in patients with PDAC. The PFS was longer in patients with a BRCA 1/2 mutation than those with a wild type, even though the difference was not statistically significant. In this study, the rate of $B R C A 1 / 2$ mutations was $13.6 \%$. The data values were slightly higher than previous data (range 4-7\%) in the general population [4,5]. The higher proportion of $B R C A 1 / 2$ mutations may be due to the change in detection method with the adoption of next-generation sequencing. In addition, considering that a high proportion of patients were previously diagnosed with breast cancer in this study, the results are similar to those of previous studies. The prevalence of BRCA 1/2 mutations in Asian patients with familial breast cancer and early-onset breast cancer was reported to be $2.8 \%$ to $31.8 \%$ [18]. Previous studies showed that BRCA gene mutations were associated with patients' survival outcomes $[12,13,19]$. In this study, patients with $B R C A$ gene mutations did not show different survival outcomes on account of the small number of patients.

The clinical significance and prognostic value of germline BRCA pathogenic mutations in tumors are well-known, but whether missense variants of uncertain significance (VUS) have clinical impact is not known. Variants in the gene were often classified as VUSs because of an insufficient understanding of the gene's role. Variants can be reclassified from VUS to likely pathogenic, and further, to pathogenic. Phosphorylation of BRCA 1/2 mutations plays an important role in their function as regulators of DNA repair, transcription, and cell cycles in response to DNA damage. Tram et al. suggested that VUS have the potential to interfere with the phosphorylation process via abolishing or creating phosphorylation sites on $B R C A 1 / 2$ [20]. Hu et al. reported that germline VUS variant carriers had superior disease-free survival when compared with wild-type PDAC patients receiving adjuvant chemotherapy (16.5 months vs. 13.1 months, $p=0.007$ ) [21]. Previous statistics indicate that between $10-20 \%$ of BRCA sequencing results are VUSs, and of these, more than $50 \%$ are missense mutations [22]. In this study, BRCA 1/2 missense mutations (VUSs) were detected in $15.2 \%$ of our cohort (Supplementary Table S1). The ORR was significantly higher in BRCA-positive patients, including missense mutations of VUS, than in BRCA-negative patients $(7 / 12,58.3 \%$ vs. $3 / 31,9.7 \%, p=0.002)$. With the further accumulation of data in the future, VUS can be reclassified as pathogenic.

Previously, several studies reported on the proportion of $B R C A 1 / 2$ mutations and their impact on patients with PDAC $[9,12,13,16,19,23-28]$. Golan et al. showed a difference in survival outcome for stage 3 or 4 PDAC patients with BRCA 1/2 mutations in platinum-based chemotherapy (22 months vs. 9 months, $p=0.039$ ) [19]. Wattenberg et al. reported on the treatment response of platinum-based chemotherapy in PDAC patients with $B R C A 1 / 2$ mutations (58\% vs. $21 \%, p=0.002$ ) [12]. In the present study, patients who received FOLFIRINOX chemotherapy showed a better treatment response in $B R C A$-positive patients compared to $B R C A$-negative patients. However, patients who received nab-paclitaxel chemotherapy did not show any difference in treatment response, irrespective of $B R C A$ mutations.

Recently, several studies attempted to identify patients who benefit from palliative first-line FOLFIRINOX chemotherapy. Transcriptomic analysis showed that the basal type showed a better treatment response to FOLFIRINOX chemotherapy. The immunohistochemistry stained marker KRT81 may be a predictive marker to identify patients in the clinical field [29]. Circulating blood markers, such as ctDNA and exosomes, were also suggested as predictors for FOLFIRINOX response [30]. In other studies, protein markers, CES2 expression, and female gender predicted the response to FOLFIRINOX in PDAC [31,32]. The ideal predictor is a non-invasive clinically feasible tool during patient treatment. In this study, BRCA 1/2 was a predictor of the response to FOLFIRINOX. However, the proportion of $B R C A$ cases was very low in patients with PDAC. Several clinical trials are currently ongoing to identify better blood germline biomarkers (ClinicalTrials.gov NCT04289961; NCT04143152). 
Despite the efficacy of $B R C A$ on treatment response in patients, the present study did not show survival benefits in patients who underwent FOLFIRINOX. Regardless of how good a prognostic or therapeutic predictive marker may be, it cannot outperform clinical parameters, such as cancer stage, age, sex, and metastasis, on their prognosis. Germline mutations can be used to predict FOLFIRINOX treatment response; however, they are still limited in predicting patient prognosis. A previous study by Sehdev et al. and Golan et al. also showed a significant difference in the prognosis of BRCA-positive patients who received platinum-based chemotherapy $[16,24]$.

Our study has strengths. This is the first report of the ORR in numerous patients with BRCA 1/2 mutations following the use of FOLFIRINOX in Asia. In a previous study, less was known about the prevalence and treatment outcomes of FOLFIRINOX involving $B R C A 1 / 2$ mutations in Asia [14]. The high ORR of $71.4 \%$ with FOLFIRINOX therapy in $B R C A$-positive patients suggests that platinum therapy may be particularly desirable for this subset of patients in clinical scenarios marked by high disease burden and symptomatic disease, and for patients with PDAC. This study may help guide treatment decisions for patients with PDAC.

This study has several limitations. First, this is a retrospective study. Although we adjusted several factors via multivariate analysis, selection and/or information bias could remain. The lack of statistically significant differences in both OS and PFS in this study population may be attributed to the limited number of patients enrolled in the study: only 32 patients in our study were treated with palliative, first-line FOLFIRINOX. Second, although we found no significant difference in the proportion of males between groups, there were fewer males in the BRCA-positive group $(2 / 9,22.2 \%$ vs. $22 / 55,40.0 \%$, $p=0.464$ ) [32]. In this study, relatively young patients were enrolled compared to previous studies (median 57.5 years) [12,19,33]. Both findings could plausibly skew bias toward the null hypothesis. Third, we were unable to control for mortality comorbidities that might have affected our results. However, since FOLFIRINOX is indicated for relatively healthier PDAC patients with good performance status, we do not think that the difference in comorbidities is the only explanation for our results.

\section{Conclusions}

We found that the presence of germline BRCA 1/2 mutations is associated with an improved ORR in PDAC patients treated with FOLFIRINOX. These results validate the association of germline $B R C A 1 / 2$ mutations with platinum sensitivity, as reported by other results in patients with PDAC. Notably, the high response rate in this analysis supports the preferential use of FOLFIRINOX therapy for patients with PDAC harboring a BRCA germline mutation, and supports the need for early germline testing in order to select the best therapy. Further prospective studies are needed to refine the treatment paradigms for this important subset of patients with PDAC.

Supplementary Materials: The following supporting information can be downloaded at: https: / / www.mdpi.com/article/10.3390/cancers14010236/s1, Figure S1: Kaplan-Meier curve for overall survival in patients stratified by presence of germline BRCA gene mutation, Table S1: Pathogenic mutations seen in 9 patients (ID) and variant of unknown significant mutations seen in 10 patients in BRCA genes of our cohort, Table S2: Univariate and multivariate analysis using the Cox regression analysis to identify the risk factors for overall survival, Table S3: Overall response rate to FOLFIRINOX in patients with a germline $B R C A 1 / 2$ mutation including variant of unknown significance was significantly higher than patients without the mutation.

Author Contributions: J.H.P. and H.S.L. had the initial idea and performed the research. J.H.P. and H.S.L. wrote the paper. J.H.J., S.I.J., M.J.C., J.Y.P., S.W.P., S.B., S.Y.S., H.S.L. and J.H.C. evaluated and suggested changes to the manuscript. H.S.L. and J.H.C. approved the final version of the manuscript. All authors have read and agreed to the published version of the manuscript.

Funding: This study was supported by a faculty research grant of the Yonsei University College of Medicine (6-2018-0082). 
Institutional Review Board Statement: The study was performed in accordance with the Declaration of Helsinki, as reflected by the institutional review board of Severance Hospital (approval number 4-2021-1151).

Informed Consent Statement: Informed consent was waived because of the retrospective nature of the study, and the analysis used anonymous clinical data.

Data Availability Statement: The data presented in this study are available on request from the corresponding author.

Conflicts of Interest: The funders had no role in the design of the study; in the collection, analyses, or interpretation of data; in the writing of the manuscript; or in the decision to publish the results.

\section{References}

1. Rahib, L.; Smith, B.D.; Aizenberg, R.; Rosenzweig, A.B.; Fleshman, J.M.; Matrisian, L.M. Projecting Cancer Incidence and Deaths to 2030: The Unexpected Burden of Thyroid, Liver, and Pancreas Cancers in the United States. Cancer Res. 2014, 74, $2913-2921$. [CrossRef] [PubMed]

2. Tempero, M.A.; Malafa, M.P.; Al-Hawary, M.; Behrman, S.W.; Benson, A.B.; Cardin, D.B.; Chiorean, E.G.; Chung, V.; Czito, B.; Del Chiaro, M.; et al. Pancreatic Adenocarcinoma, Version 2.2021, NCCN Clinical Practice Guidelines in Oncology. J. Natl. Compr. Cancer Netw. 2021, 19, 439-457. [CrossRef] [PubMed]

3. Zhu, H.; Wei, M.; Xu, J.; Hua, J.; Liang, C.; Meng, Q.; Zhang, Y.; Liu, J.; Zhang, B.; Yu, X.; et al. PARP inhibitors in pancreatic cancer: Molecular mechanisms and clinical applications. Mol. Cancer 2020, 19, 49. [CrossRef]

4. Iqbal, J.; Ragone, A.; Lubinski, J.; Lynch, H.T.; Moller, P.; Ghadirian, P.; Foulkes, W.D.; Armel, S.; Eisen, A.; Neuhausen, S.L.; et al. The incidence of pancreatic cancer in BRCA1 and BRCA2 mutation carriers. Br. J. Cancer 2012, 107, 2005-2009. [CrossRef] [PubMed]

5. Holter, S.; Borgida, A.; Dodd, A.; Grant, R.; Semotiuk, K.; Hedley, D.; Dhani, N.; Narod, S.; Akbari, M.; Moore, M.; et al. Germline BRCA Mutations in a Large Clinic-Based Cohort of Patients with Pancreatic Adenocarcinoma. J. Clin. Oncol. 2015, 33, 3124-3129. [CrossRef] [PubMed]

6. John, E.M.; Hopper, J.L.; Beck, J.C.; Knight, J.A.; Neuhausen, S.L.; Senie, R.T.; Ziogas, A.; Andrulis, I.L.; Anton-Culver, H.; Boyd, N.; et al. The Breast Cancer Family Registry: An infrastructure for cooperative multinational, interdisciplinary and translational studies of the genetic epidemiology of breast cancer. Breast Cancer Res. 2004, 6, R375. [CrossRef]

7. Hahn, S.A.; Greenhalf, B.; Ellis, I.; Sina-Frey, M.; Rieder, H.; Korte, B.; Gerdes, B.; Kress, R.; Ziegler, A.; Raeburn, J.A.; et al. BRCA2 germline mutations in familial pancreatic carcinoma. J. Natl. Cancer Inst. 2003, 95, 214-221. [CrossRef] [PubMed]

8. Golan, T.; Hammel, P.; Reni, M.; Van Cutsem, E.; Macarulla, T.; Hall, M.J.; Park, J.O.; Hochhauser, D.; Arnold, D.; Oh, D.Y.; et al Maintenance Olaparib for Germline BRCA-Mutated Metastatic Pancreatic Cancer. N. Engl. J. Med. 2019, 381, 317-327. [CrossRef]

9. Navarro, E.B.; Lopez, E.V.; Quijano, Y.; Caruso, R.; Ferri, V.; Durand, H.; Cabrera, I.F.; Reques, E.D.; Ielpo, B.; Glagolieva, A.Y.; et al. Impact of BRCA 1/2 gene mutations on survival of patients with pancreatic cancer: A case-series analysis. Ann. Hepato-BiliaryPancreat. Surg. 2019, 23, 200-205. [CrossRef]

10. Turner, N.; Tutt, A.; Ashworth, A. Hallmarks of 'BRCAness' in sporadic cancers. Nat. Rev. Cancer 2004, 4, 814-819. [CrossRef]

11. Husain, A.; He, G.; Venkatraman, E.S.; Spriggs, D.R. BRCA1 up-regulation is associated with repair-mediated resistance to cis-diamminedichloroplatinum(II). Cancer Res. 1998, 58, 1120-1123. [PubMed]

12. Wattenberg, M.M.; Asch, D.; Yu, S.; O’Dwyer, P.J.; Domchek, S.M.; Nathanson, K.L.; Rosen, M.A.; Beatty, G.L.; Siegelman, E.S.; Reiss, K.A. Platinum response characteristics of patients with pancreatic ductal adenocarcinoma and a germline BRCA1, BRCA2 or PALB2 mutation. Br. J. Cancer 2020, 122, 333-339. [CrossRef]

13. Golan, T.; Barenboim, A.; Lahat, G.; Nachmany, I.; Goykhman, Y.; Shacham-Shmueli, E.; Halpern, N.; Brazowski, E.; Geva, R.; Wolf, I.; et al. Increased Rate of Complete Pathologic Response after Neoadjuvant FOLFIRINOX for BRCA Mutation Carriers with Borderline Resectable Pancreatic Cancer. Ann. Surg. Oncol. 2020, 27, 3963-3970. [CrossRef] [PubMed]

14. Shimmura, H.; Kuramochi, H.; Jibiki, N.; Katagiri, S.; Nishino, T.; Araida, T. Dramatic response of FOLFIRINOX regimen in a collision pancreatic adenocarcinoma patient with a germline BRCA2 mutation: A case report. Jpn. J. Clin. Oncol. 2019, 49, 1049-1054. [CrossRef]

15. Rebelatto, T.F.; Falavigna, M.; Pozzari, M.; Spada, F.; Cella, C.A.; Laffi, A.; Pellicori, S.; Fazio, N. Should platinum-based chemotherapy be preferred for germline BReast CAncer genes (BRCA) 1 and 2-mutated pancreatic ductal adenocarcinoma (PDAC) patients? A systematic review and meta-analysis. Cancer Treat. Rev. 2019, 80, 101895. [CrossRef] [PubMed]

16. Sehdev, A.; Gbolahan, O.; Hancock, B.A.; Stanley, M.; Shahda, S.; Wan, J.; Wu, H.H.; Radovich, M.; O’Neil, B.H. Germline and Somatic DNA Damage Repair Gene Mutations and Overall Survival in Metastatic Pancreatic Adenocarcinoma Patients Treated with FOLFIRINOX. Clin. Cancer Res. 2018, 24, 6204-6211. [CrossRef]

17. Kim, B.; Won, D.; Jang, M.; Kim, H.; Choi, J.R.; Kim, T.I.; Lee, S.T. Next-generation sequencing with comprehensive bioinformatics analysis facilitates somatic mosaic APC gene mutation detection in patients with familial adenomatous polyposis. BMC Med. Genom. 2019, 12, 103. [CrossRef] 
18. Kim, H.; Choi, D.H. Distribution of BRCA1 and BRCA2 Mutations in Asian Patients with Breast Cancer. J. Breast Cancer 2013, 16, 357-365. [CrossRef]

19. Golan, T.; Kanji, Z.S.; Epelbaum, R.; Devaud, N.; Dagan, E.; Holter, S.; Aderka, D.; Paluch-Shimon, S.; Kaufman, B.; GershoniBaruch, R.; et al. Overall survival and clinical characteristics of pancreatic cancer in BRCA mutation carriers. Br. J. Cancer 2014, 111, 1132-1138. [CrossRef]

20. Tram, E.; Savas, S.; Ozcelik, H. Missense variants of uncertain significance (VUS) altering the phosphorylation patterns of BRCA1 and BRCA2. PLOS ONE 2013, 8, e62468. [CrossRef]

21. Hu, H.; Zhu, Y.; Pu, N.; Burkhart, R.A.; Burns, W.; Laheru, D.; Zheng, L.; He, J.; Goggins, M.G.; Yu, J. Association of Germline Variants in Human DNA Damage Repair Genes and Response to Adjuvant Chemotherapy in Resected Pancreatic Ductal Adenocarcinoma. J. Am. Coll. Surg. 2020, 231, 527-535.e514. [CrossRef]

22. Jimenez-Sainz, J.; Jensen, R.B. Imprecise Medicine: BRCA2 Variants of Uncertain Significance (VUS), the Challenges and Benefits to Integrate a Functional Assay Workflow with Clinical Decision Rules. Genes 2021, 12, 780. [CrossRef]

23. Lowery, M.A.; Kelsen, D.P.; Stadler, Z.K.; Yu, K.H.; Janjigian, Y.Y.; Ludwig, E.; D' Adamo, D.R.; Salo-Mullen, E.; Robson, M.E.; Allen, P.J.; et al. An emerging entity: Pancreatic adenocarcinoma associated with a known BRCA mutation: Clinical descriptors, treatment implications, and future directions. Oncologist 2011, 16, 1397-1402. [CrossRef]

24. Golan, T.; Sella, T.; O’Reilly, E.M.; Katz, M.H.; Epelbaum, R.; Kelsen, D.P.; Borgida, A.; Maynard, H.; Kindler, H.; Friedmen, E.; et al. Overall survival and clinical characteristics of BRCA mutation carriers with stage I/II pancreatic cancer. Br. J. Cancer 2017, 116, 697-702. [CrossRef]

25. Gostimir, M.; Bennett, S.; Moyana, T.; Sekhon, H.; Martel, G. Complete pathological response following neoadjuvant FOLFIRINOX in borderline resectable pancreatic cancer-A case report and review. BMC Cancer 2016, 16, 786. [CrossRef]

26. Vyas, O.; Leung, K.; Ledbetter, L.; Kaley, K.; Rodriguez, T.; Garcon, M.C.; Saif, M.W. Clinical outcomes in pancreatic adenocarcinoma associated with BRCA-2 mutation. Anticancer Drugs 2015, 26, 224-226. [CrossRef] [PubMed]

27. Zhao, Q.; Rashid, A.; Gong, Y.; Katz, M.H.; Lee, J.E.; Wolf, R.; Balachandran, A.; Varadhachary, G.R.; Pisters, P.W.; Wang, H.; et al Pathologic complete response to neoadjuvant therapy in patients with pancreatic ductal adenocarcinoma is associated with a better prognosis. Ann. Diagn. Pathol. 2012, 16, 29-37. [CrossRef]

28. Sonnenblick, A.; Kadouri, L.; Appelbaum, L.; Peretz, T.; Sagi, M.; Goldberg, Y.; Hubert, A. Complete remission, in BRCA2 mutation carrier with metastatic pancreatic adenocarcinoma, treated with cisplatin based therapy. Cancer Biol. 2011, 12, 165-168. [CrossRef] [PubMed]

29. Muckenhuber, A.; Berger, A.K.; Schlitter, A.M.; Steiger, K.; Konukiewitz, B.; Trumpp, A.; Eils, R.; Werner, J.; Friess, H.; Esposito, I.; et al. Pancreatic Ductal Adenocarcinoma Subtyping Using the Biomarkers Hepatocyte Nuclear Factor-1A and Cytokeratin-81 Correlates with Outcome and Treatment Response. Clin. Cancer Res. 2018, 24, 351-359. [CrossRef]

30. van der Sijde, F.; Vietsch, E.E.; Mustafa, D.A.M.; Besselink, M.G.; Groot Koerkamp, B.; van Eijck, C.H.J. Circulating Biomarkers for Prediction of Objective Response to Chemotherapy in Pancreatic Cancer Patients. Cancers 2019, 11, 93. [CrossRef] [PubMed]

31. Capello, M.; Lee, M.; Wang, H.; Babel, I.; Katz, M.H.; Fleming, J.B.; Maitra, A.; Wang, H.; Tian, W.; Taguchi, A.; et al. Carboxylesterase 2 as a Determinant of Response to Irinotecan and Neoadjuvant FOLFIRINOX Therapy in Pancreatic Ductal Adenocarcinoma. J. Natl. Cancer Inst. 2015, 107, djv132. [CrossRef] [PubMed]

32. Hohla, F.; Hopfinger, G.; Romeder, F.; Rinnerthaler, G.; Bezan, A.; Stättner, S.; Hauser-Kronberger, C.; Ulmer, H.; Greil, R. Female gender may predict response to FOLFIRINOX in patients with unresectable pancreatic cancer: A single institution retrospective review. Int. J. Oncol. 2014, 44, 319-326. [CrossRef] [PubMed]

33. Conroy, T.; Desseigne, F.; Ychou, M.; Bouche, O.; Guimbaud, R.; Becouarn, Y.; Adenis, A.; Raoul, J.L.; Gourgou-Bourgade, S.; de la Fouchardiere, C.; et al. FOLFIRINOX versus gemcitabine for metastatic pancreatic cancer. N. Engl. J. Med. 2011, 364, 1817-1825. [CrossRef] [PubMed] 